\title{
An image enhancement method based on gabor filtering in wavelet domain and adaptive histogram equalization
}

\author{
Jeevan K M, Anne Gowda A B, Padmaja Vijay Kumar
}

GITAM School of Technology, GITAM Deemed to be University, Bangalore, India

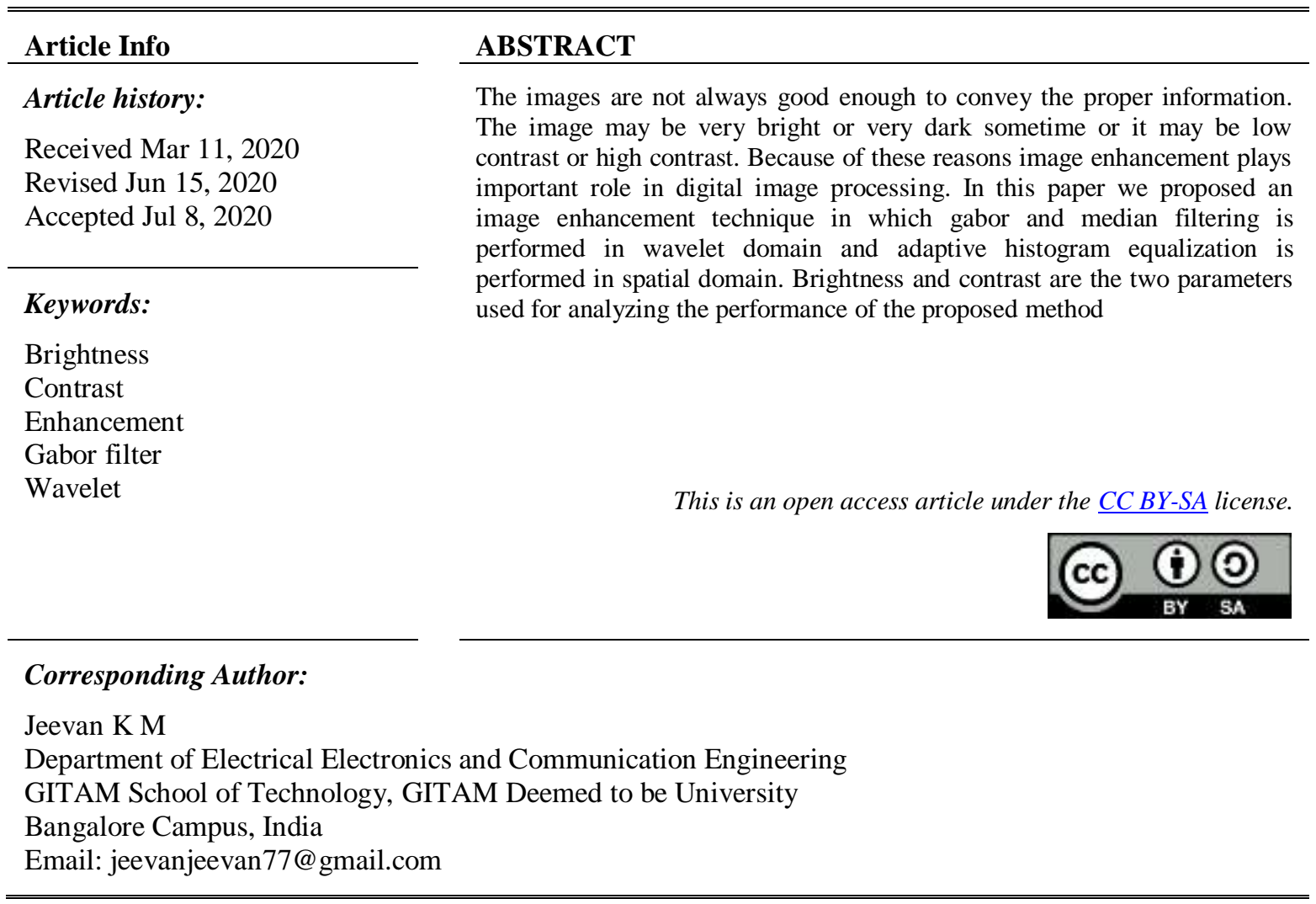

\section{INTRODUCTION}

When we present the output of an imaging system to a human, we should consider how the output is transferred into information by the observer. That is we need to consider the property of human visual perception. Visual perception may vary person to person. Two important parameters related to human visual perception are brightness and contrast. Brightness of an image is considered as the overall darkness or lightness of the image. It depends on our visual perception. Contrast is the word used to emphasize the difference in grey level of the image. Difference between maximum pixel intensity and minimum pixel intensity of an image is termed as contrast.

Depending on the brightness and contrast an image can be a dark, bright, low contrast or high contrast image. By visual inspection, one can identify the nature of the image. We can also identify the nature of the image using its histogram. The histogram provides the number of occurrences of particular gray level in the image. The histogram is clustered towards low side of the gray scale for a dark image and for a bright image it is clustered towards high side of the gray scale. In case of low contrast image, the histogram is narrow and centered towards the middle of the gray scale. The histogram covers the broad range of the gray scale for a high contrast image.

We may have a dark image or brighter image depending on the situation when we capture the image. The dark image is due to under exposed condition and brighter image is due to over exposed condition. The image with low contrast is a result of poor lighting, low dynamic range of sensors which are used for acquiring the images or wrong setting of aperture of lens. This indicates the image is not perfect sometime. 
Therefore, in order to identify all the information present in an image, image enhancement is required. In this paper we describe a method for enhancing darker images.

The rest of the paper is organized as follows. The Section 2 is literature review, which describes latest state of the art methods used for image enhancement. In Section 3 the proposed method for image enhancement is explained. Result and discussions, and conclusions are given in Section 4 and 5 respectively.

\section{LITERATURE SURVEY}

Image enhancement is the way toward modifying digital images with the goal that the outcomes are increasingly appropriate for show or further image analysis. Image enhancement has seen as presumably the most significant vision applications since it has capacity to upgrade the perceivability of pictures. It improves the detectable quality of poor pictures. Particular systems have been proposed therefore for improving the nature of the advanced pictures. To upgrade picture quality image enhancement can specifically improve and restrain a few information offered inside the input image. The premier reason for applying enhancement techniques is to bring out the hidden details in an image or to improve the contrast. It gives a large number of determinations to bettering the visible quality of images. Enhancement method can be classified into spatial domain methods and Transform domain methods.

Histogram equalization (HE) is a method for changing image intensities to enhance contrast. The histogram equalization is mainly used for enhancing the global contrast of images. This method is effective when the usable information is represented by close contrast values in the images. Through this adjustment, the intensities can be better dispersed on the histogram. This takes into account regions of lower local contrast to increase a higher contrast. Even though $\mathrm{HE}$ is computationally efficient, this method provides too much enhancement. It also gives abnormal artifacts on the images.

Another effective method for image enhancement is adaptive histogram equalization (AHE). In AHE, different histograms are identified from the image, each corresponding to different section of the image. The enhancement is done by redistributing the probability density of pixel values of each section. Because of this, AHE is suitable for enhancing the local contrast. In AHE, there is a possibility of over enhancing the contrast in near contrast region. Therefore, such regions will be highly concentrated after enhancement process. It is due to this, noise also may get amplified in near contrast region. This is one disadvantage of AHE.

Following section describes some state-of-the-art works done in image enhancement. Burak YILMAZ, Yu"ksel OZBAY [1] proposed a method for enhancing the contrast of the image using linear image combinations algorithm. In order to improve the quality of medical images Hammid Hassanpour et al [2] proposed a method based on morphological transform. They proposed a filter based on Top-Hat transform and which is applied to the image for getting enhance image.

Han-Yen Tu et al [3] proposed an efficient adaptive image enhancement (AIE) Method in Wavelet Domain for Handwritten Document. An AIE method is proposed to denoise handwritten documents in a wavelet domain, which differs from other methods in two aspects. Firstly, modified contrast limited adaptive histogram equalization (MCLAHE) is used to equalize the contrast of an image by cutting the histogram at some threshold, and then equalization is used. Secondly, the image is improved by using directional discrete wavelet transform (D2WT) enhancing for foreground and interfering strokes, respectively.

He Wen, Wu Qi and Li Shuang [4] prblished paper for X-ray image enhancement. They proposed a method using Homomorphic filter. The filtering is performed in wavelet domain. The image to be enhanced is decomposed into low and high frequency components using wavelet transform. The filtering is done in low frequency band and the high frequency bands are processed by wavelet thresholding. As a final step, contrast limited adaptive histogram equalization is applied on the wavelet reconstructed image.

G. Padma Priya and T. Venkateswarlu [5] proposed a modified algorithm, through which better performance can be achieved for low contrast images with low and mid intensities. The proposed technique considers the weighted sum of singular value matrices of LL sub band image of low contrast image and LL sub band image of General Histogram Equalized image. The proposed method is compared with general histogram equalization (GHE), contrast-limited adaptive histogram equalization (CLAHE) and existing DWT-SVD methods.

Zhang Qinli et al [6] proposed a novel medical image enhancement method based on wavelet decomposition by adding details from the high-frequency sub-images and decomposing the image, specially with anti-symmetric biorthogonal wavelet instead of some traditional wavelets. It not only improves the image enhancement, but also overcomes the shortcomings of large computation with faster computational speed and satisfies the real-time requirement in edge detection.

An adaptive pixel value stretching based image enhancement method was proposed by Gang Cao et al [7]. In this method, the saturation for each pixels are set by perceptual brightness of global image. A figure 
print enhancement method was proposed by Erwin et al [8]. In this method a combination of Gabor filter and AHE was used. Application of Gabor filter in image enhancement is also discussed in [9, 10]. Some other important enhancement techniques and comparitive study of different image enhancement methods are available in [11-21].

\section{RESEARCH METHOD}

This section illustrates the proposed method for enhancement of dark image. In this method the combination of adaptive histogram equalization (AHE), Gabor filtering and median filtering is used for enhancement. AHE is performed in spatial domain and, Gabor and median filtering is done in wavelet domain.

\subsection{Problem statement}

Let $I=\{f(x, y) \mid i=1,2, \ldots \ldots R ; j=1,2, \ldots \ldots . C\}$ be a grayscale image with $\mathrm{R} X \mathrm{C}$ pixels, where $\mathrm{R}$ and $\mathrm{C}$ are the number of rows and column respectively and $\mathrm{f}(\mathrm{x}, \mathrm{y})=0,1,2 \ldots .255$ for an 8 -bit image. In many cases, the captured image 'I' may not have suitable brightness or some time the dynamic range may be limited. It is very difficult to understand the image detail from such images. For example, if we capture the image in under exposed condition, it will be too dark to display the content clearly. In this scenario, the objective of our work is to develop an image enhancement algorithm for very dark images.

\subsection{Methodology}

AHE is an excellent method for contrast enhancement. This method is useful for the contrast enhancement of natural images as well as medical images. However, two main problems are associated with AHE. They are low speed and over enhancement of noises [22]. Therefore, in order to get a better enhanced image with reduced noises, in the proposed method we introduced Gabor filtering [8] and median filtering along with AHE. The block diagram shown in Figure 1 illustrates the steps followed in the proposed method.

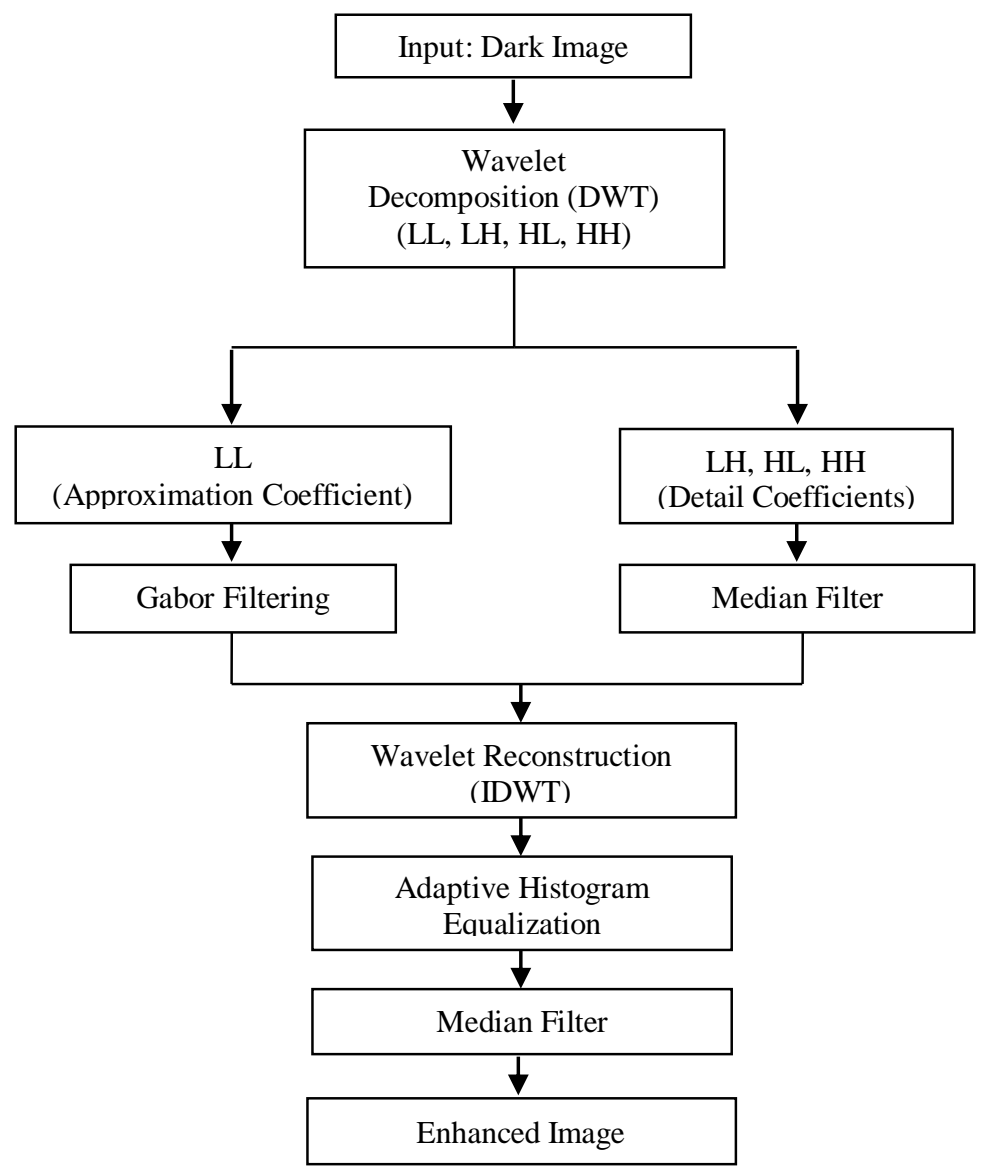

Figure 1. Block diagram of proposed method for image enhancement 
The proposed method is as follows. The dark image is first decomposed to approximation (LL) and detail (LH, HL, HH) coefficients using Haar wavelet. Different type of wavelet exist and among them Haar wavelet is the simplest and shortest [23, 24]. We tested our algorithm with different orthogonal wavelet families, but it was found that Haar wavelet is best suitable for edge increasing. Applying the Haar wavelet limits the impacts of the relationship disturbance among components, and makes a small buffer around the edge. Because of these reasons we selected Haar wavelet for decomposition.

The wavelet decomposition results in approximation and detail components. We applied Gabor filtering in approximation (LL) and median filtering in detail ( $\mathrm{LH}, \mathrm{HL}, \mathrm{HH})$ components. Orientation selectivity is the main feature of Gabor filter. In the spatial domain, a 2D Gabor filter is a Gaussian kernel function modulated by a sinusoidal plane wave [25, 26]. As shown in (1) represents the formula for 2DGabor filter in spatial domain

$$
G(x, y ; \theta, f)=\exp \left\{-\frac{1}{2}\left[\frac{x_{\theta}^{2}}{\sigma_{x}^{2}}+\frac{y_{\theta}^{2}}{\sigma_{y}^{2}}\right]\right\} \cos \left(2 \pi f x_{\theta}\right)
$$

where $x_{\theta}=x \cos \theta-y \sin \theta$ andy $y_{\theta}=x \sin \theta+y \cos \theta, \theta$ is the orientation direction, $f$ is the $\operatorname{cosine}$ wave frequency $\square \mathrm{x}$ and $\square \mathrm{y}$ is a fixed distance from the Gaussian properties respectively along the $\mathrm{x}$ and $\mathrm{y}$-axes.

Median filter is a non linear filter which replaces the value of a pixel with the median of the values of the neighborhood of that pixel. The median filter compute the median of the corrupted image $g(x, y)$ in the area defined by Sxy. The value of the filtered image $f$ at any point $(x, y)$ is the median of the pixels in the region defined by Sxy. It can be represented as given in (2).

$$
f(x, y)^{\wedge}=\operatorname{median}\{g(s, t)\},(s, t) \in S_{x y}
$$

The LH, HL, and HH band comprise of high frequency parts. The high frequency components may represents some noises and edges in the image. The median filter gives considerably less blurring than mean filter and it preserve the edges. As a result of these reasons we utilized median filter, in this work, for filtering detail components.

After applying Gabor filtering to $\mathrm{LL}$ and median filtering to $\mathrm{LH}, \mathrm{HL}$ and $\mathrm{HH}$, the image is reconstructed using inverse discrete wavelet transform (IDWT). That is, now we have the image in spatial domain. This image is then enhanced using AHE. As we discussed earlier, the noises also enhanced by AHE. In order to reduce these noises, we applied median filtering once again.

\section{RESULTS AND DISCUSSION}

Image enhancement is one of the important areas in image processing. The captured images are not always good enough to apply the image processing algorithm directly. The image may not have good brightness or contrast some time. So in order to adjust the brightness or contrast, we have to perform image enhancement before applying other image processing techniques. In this paper, an enhancement method which is the combination of spatial and transformed domain is proposed.

The method is simulated using MATLAB. Figure 2 and 3 shows the simulation results of two different images. From the figure (Figure 2(d) and 3(d)) it is identified that the histogram of dark images is concentrated towards the low values of gray level and the histogram of the images after applying the proposed method (Figure 2(f) and 3(f)) is spread over wide range of gray scale. By analyzing the histogram, it is identified that the proposed method gives better enhancement.

The performance of the proposed method is analyzed using the parameters brightness and contrast. Brightness can be considered as an element of human visual perception. It indicates luminance of a visual target. The brightness of each pixel in the image is its gray level value and therefore the intensity of the image can be calculated as the mean of the grey level values. As shown in (3) is used for finding the brightness (B).

$$
B=\frac{1}{M N} \sum_{i=1}^{M} \sum_{j=1}^{N} I(i, j)
$$

Contrast can be explained as the difference between maximum and minimum grey level value in an image. In this work, we used RMS contrast as given in the (4), for the performance analysis.

$$
C=\sqrt{\frac{1}{M N} \sum_{i=1}^{M} \sum_{j=1}^{N}[(I(i, j)-B)]^{2}}
$$

where B is the brightness, determined using (3). 
Figure 2 and 3 depicts the results obtained for two different images. From the figures it is clear that the proposed method gives better results. This can be identified by analyzing the histogram. The quantitative analysis for the proposed method is done using the parameters brightness (B) and contrast (C) mentioned in (3) and (4). The results obtained for different images are tabulated in Table 1. The quantitative analysis also shows, the proposed method is better than AHE.

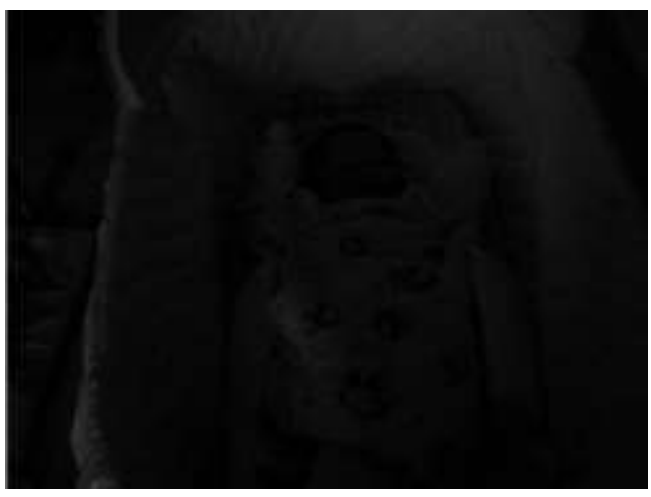

(a)

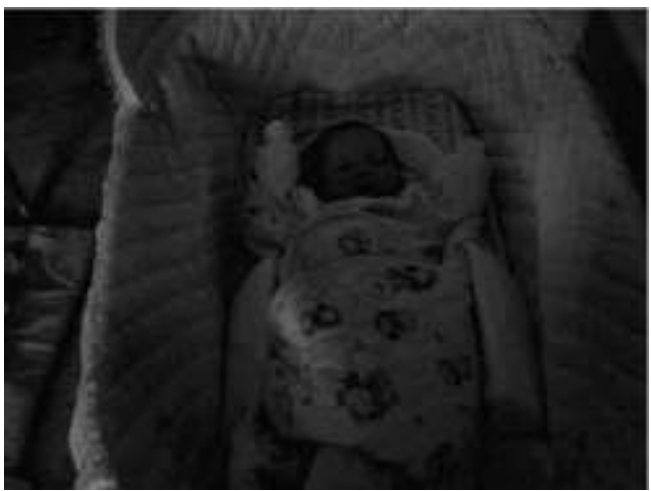

(b)

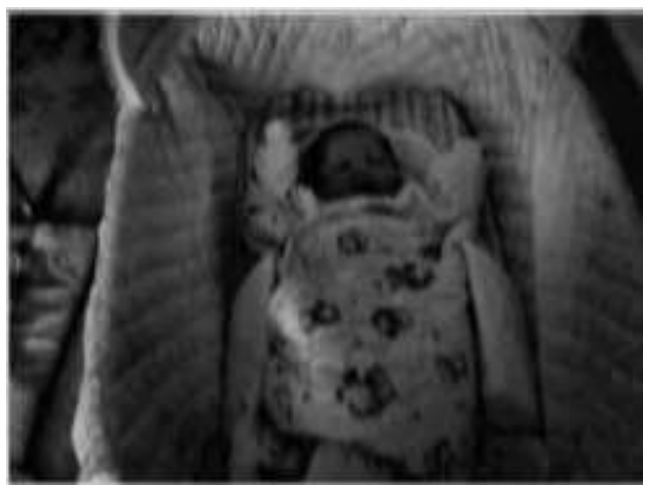

(c)

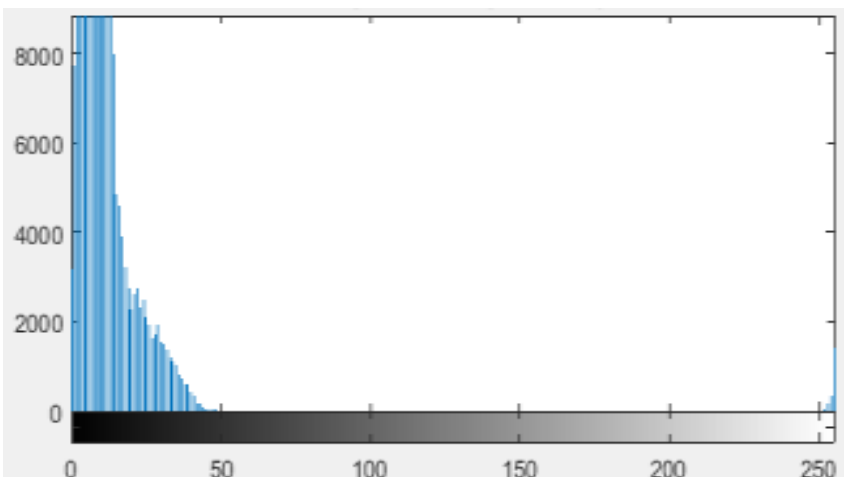

(d)

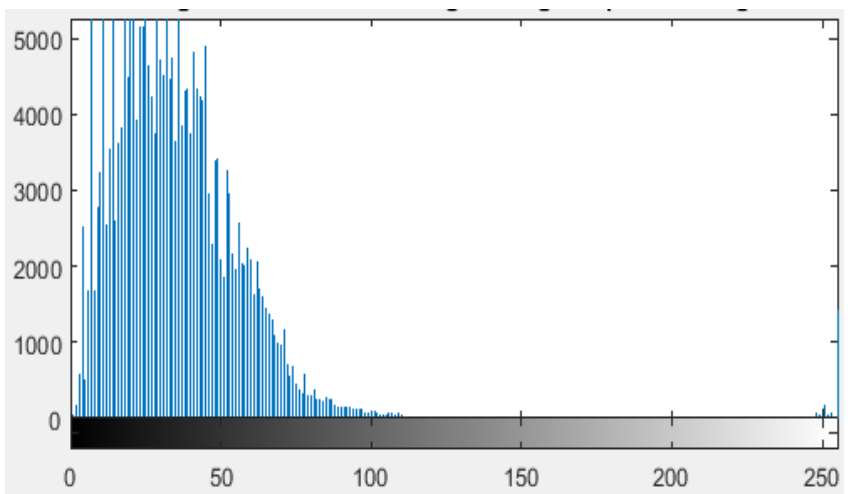

(e)

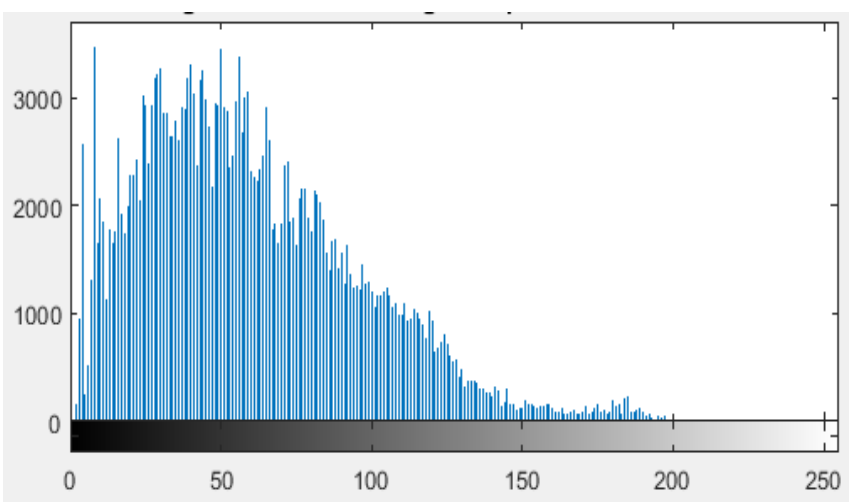

(f)

Figure 2. (a) Dark image, (b) Enhanced image using AHE, (c) Enhanced image using proposed method, (d) Histogram of dark image, (e) Histogram of enhanced image using AHE,

(f) Histogram of enhanced image using proposed method 


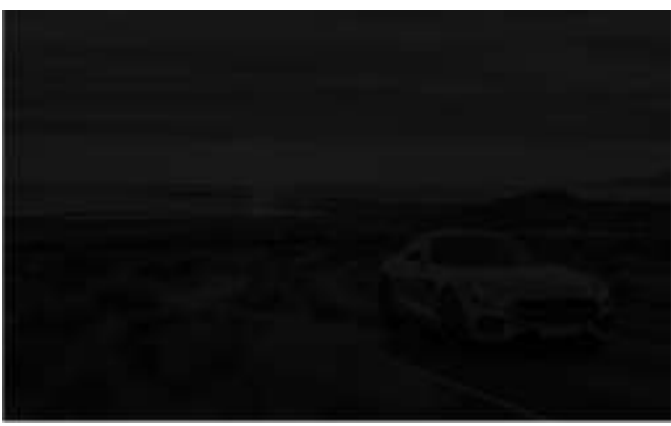

(a)

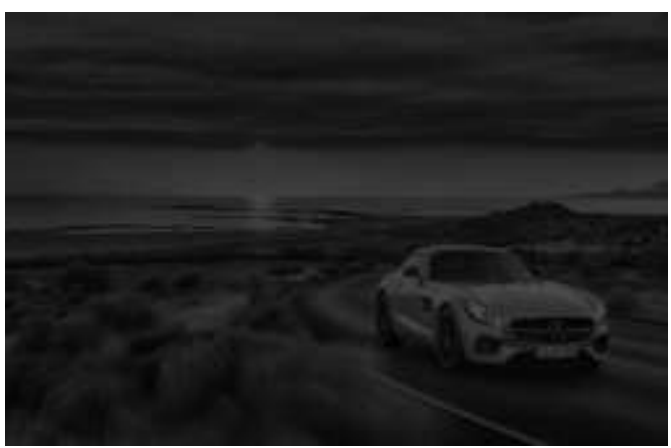

(b)

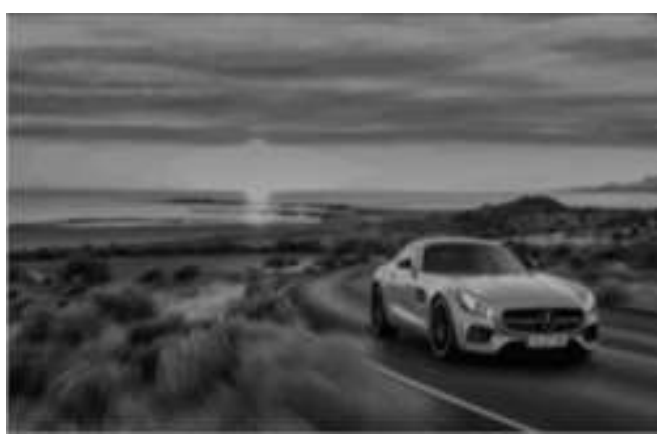

(c)

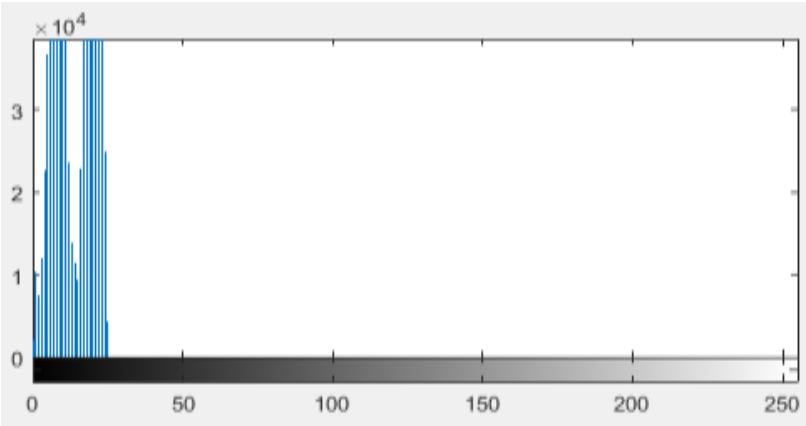

(d)

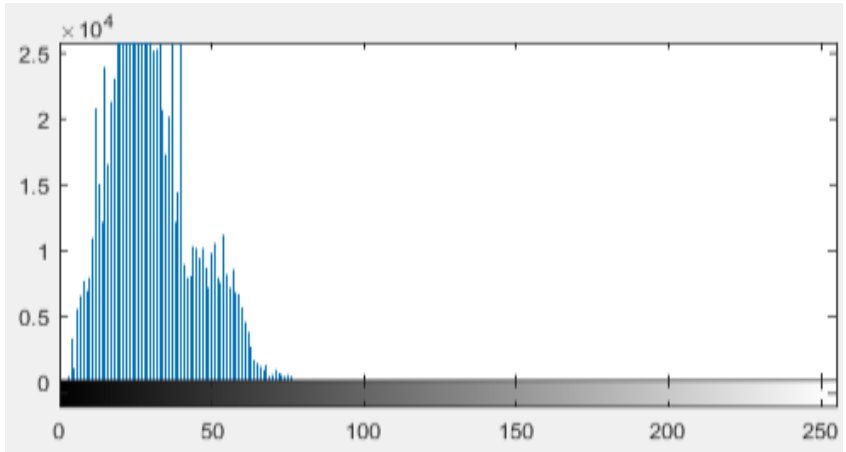

(e)

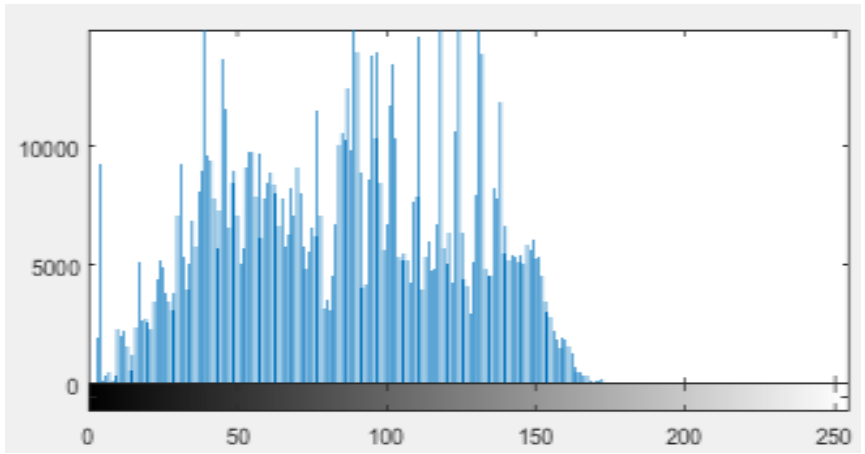

(f)

Figure 3. (a) Dark image, (b) Enhanced image using AHE, (c) Enhanced image using proposed method, (d) Histogram of dark image, (e) Histogram of enhanced image using AHE,

(f) Histogram of enhanced image using proposed method

Table1. Brightness and contrast of original image, image after AHE and proposed method

\begin{tabular}{ccccccc}
\hline \multirow{2}{*}{ Test Images } & \multicolumn{2}{c}{ Original Image } & \multicolumn{2}{c}{ AHE } & \multicolumn{2}{c}{ Proposed Method } \\
& Brightness & Contrast & Brightness & Contrast & Brightness & Contrast \\
\hline Image 1 & 12.9231 & 5.1459 & 35.7735 & 7.8903 & 60.3651 & 9.3722 \\
Image 2 & 14.0250 & 4.5818 & 29.9560 & 7.1896 & 85.9839 & 10.3010 \\
Image 3 & 13.0347 & 2.6497 & 35.3886 & 7.9462 & 92.4533 & 10.2012 \\
\hline
\end{tabular}

\section{CONCLUSION}

In this work, an image enhancement technique which is the combination of spatial domain and wavelet domain has been proposed and analyzed. The enhancement is performed in two different sections. In the first section the Gabor filtering and median filtering is performed in wavelet domain. The filtered image is then converted to time domain and AHE is performed in the second section. Both quantitative analysis and visual inspection shows that the proposed image enhancing method gives better results. 


\section{REFERENCES}

[1] B. Yilmaz and Y. Ozbay, "Contrast enhancement using linear image combinations algorithm (CEULICA) for enhancing brain magnetic resonance images," Turkish Journal of Electrical Engineering \& Computer Sciences, vol. 22, pp. 1540-1563, 2014.

[2] H. Hassanpour, et al., "Using morphological transforms to enhance the medical images," The Egyptian Journal of Radiology and Nuclear Medicine, vol. 46, pp. 481-489, 2015.

[3] H. Y. Tu, et al., "An Efficient Adaptive Image Enhancement Method in Wavelet Domain for Handwritten Document," Journal of Applied Science and Engineering, vol. 19, pp. 357-370, 2016.

[4] H. Wen, et al., "Medical X-ray Image Enhancement Based on Wavelet Domain Homomorphic Filtering and CLAHE," International Conference on Robots \& Intelligent System, pp. 249-254, 2016.

[5] G. P. Priya and T. Venkateswarlu, "Image Contrast Enhancement in Wavelet Domain," Advances in Computational Sciences and Technology, vol. 10, pp. 1915-1922, 2017.

[6] Z. Qinlia, et al., "A Novel Method of Medical Image Enhancement Based on Wavelet Decomposition," Automatic Control and Computer Sciences, vol. 51, no. 4, pp. 263-269, 2017.

[7] G. Cao, et al., "Fast contrast enhancement by adaptive pixel value stretching," International Journal of Distributed Sensor Networks, vol. 14, no. 8, pp. 1-18, 2018.

[8] Erwin, et al., "The Enhancement of Fingerprint Images using Gabor Filter," Journal of Physics: Conference Series, vol. 1196, p. 012045, 2019.

[9] P. Pakutharivu and M. V. Srinath, "Analysis of Fingerprint Image Enhancement Using Gabor Filtering with Different Orientation Field Values," Indonesian Journal of Electrical Engineering and Computer Science, vol. 5, no. 2, pp. 427-432, 2017.

[10] B. Ismail and O. Z. Mohamed, "Finger Vein Image Enhancement Technique based on Gabor filter and Discrete Cosine Transform," Journal of Telecommunication, Electronic and Computer Engineering, vol. 11, no. 2, pp. 43-48, 2019.

[11] N. Karimimehr, et al., "Fingerprint Image Enhancement Using Gabor Wavelet Transform," Proceedings of Iranian Conference on Electrical Engineering, pp. 316-320, 2010.

[12] L. S. S. Singh, et al., "A Review on Image Enhancement Methods on Different Domains," International Journal of Engineering Inventions, vol. 6, no. 1, pp. 49-55, 2017.

[13] G. B. Iwasokun and O. C. Akinyokun, "Image Enhancement Methods: A Review," British Journal of Mathematics \& Computer Science, vol. 4, no. 16, pp. 2251-2277, 2014.

[14] Y. Xie, et al., "Image Enhancement Based on Histogram Equalization," Journal of Physics: Conference Series, vol. 1314, p. 012161, 2019.

[15] B. Usman and S. Ayuba, "Practical Digital Image Enhancements using Spatial and Frequency Domains Techniques," International Research Journal of Computer Science, vol. 2, no. 5, pp. 27-32, 2015.

[16] J. Lee, et al., "An Adaptive Histogram Equalization Based Local Technique for Contrast Preserving Image Enhancement," International Journal of Fuzzy Logic and Intelligent Systems, vol. 15, no. 1, pp. 35-44, 2015.

[17] Z. Gu, et al., "A Low-Light Image Enhancement Method Based on Image Degradation Model and Pure Pixel Ratio Prior," Mathematical Problems in Engineering, vol. 2018, pp. 1-19, 2018.

[18] G. Maragatham and S. M. M. Roomi, "A Review of Image Contrast Enhancement Methods and Techniques," Research Journal of Applied Sciences, Engineering and Technology, vol. 9, no. 5, pp. 309-326, 2015.

[19] I. A. Humied and F. E. Z. Abou-Chadi, "Image Contrast Enhancement Techniques: A Comparative Study of Performance," International Journal of Computer Applications Foundation of Computer Science (FCS), vol. 137, no. 13, pp. 43-48, 2016.

[20] S. Dhariwal, "Comparative Analysis of Various Image Enhancement Techniques," International Journal of Electronics \& Communication Technology, vol. 2, no. 3, pp. 91-95, 2011.

[21] K. Akila, et al., "Mammographic image enhancement using indirect contrast enhancement techniques: A comparative study," Procedia Computer Science, vol. 47, pp. 255-261, 2015.

[22] S. M. Pizer, et al., "Adaptive Histogram Equalization and its Variations," Computer vision, Graphics and Image Processing, vol. 39, pp. 355-368, 1986.

[23] I. Daubechies, “Ten Lectures on Wavelets,” SIAM, Philadelphia, PA, USA, 1992.

[24] K. Pyka, "Wavelet-Based Local Contrast Enhancement for Satellite, Aerial and CloseRange Images," Remort Sensing, vol. 9, no. 25, pp. 1-17, 2017.

[25] https://en.wikipedia.org/wiki/Gabor_filter

[26] J. G. Daugman, "Uncertainty relations for resolution in space, spatial frequency, and orientation optimized by twodimensional visual cortical filters," Journal of the Optical Society of America, vol. 2, pp. 1160-1169, 1985. 


\section{BIOGRAPHIES OF AUTHORS}

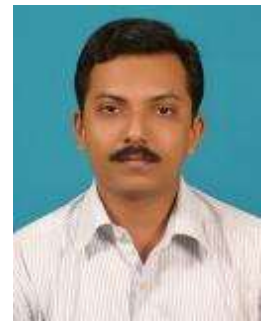

Jeevan K. M obtained his Bachelor of Technology in Electronics and Communication Engineering from Rajiv Gandhi Institute of Technology, Kottayam, Kerala, India in 1999 and Master of Technology in Electronics and Communication from College of Engineering, Trivandrum, Kerala, India in 2002. He was awarded with Ph.D. in Image Processing in 2019. He worked as a research fellow in Centre for Development of Advanced Computing (C-DAC), Trivandrum, Kerala, India and as a Hardware Design Engineer in many Multinational Companies. Currently he is associated with the department of Electronics and Communication in GITAM Deemed to be university, Bangalore, India. He has published papers in international journals and presented papers in national and international conferences. His areas of interest include signal processing and image processing.

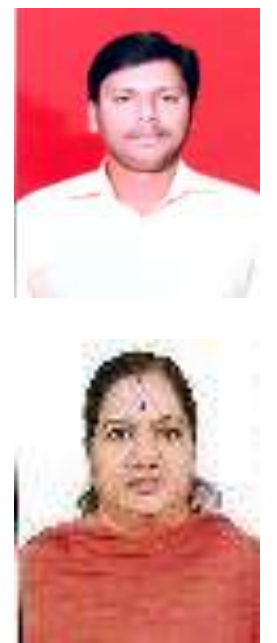

Anne Gowda A. B completed his B.Tech in Telecommunication Engineering in 2011 and M.Tech in Signal Processing in 2014. He has more than 6 years of experience in teaching. Now he is pursuing his $\mathrm{PhD}$. in Bio-Medical image processing. His area of interest includes Digital Signal Processing and Digital Image Processing.

Padmaja Vijay Kumar completed her B.E in Electronics and Communication Engineering Osmania University Hyderabad, in 1989 and M.Tech in VLSI design and embedded system from Visveswaraiah Technological University, Karnataka, India in 2006. She completed her PhD. In Image Processing in 2019 in JNTU, Government University. She had more than 25 years of teaching and research experience, (21 years in teaching and 3 years in R and D department in Industry). Her areas of interest include signal processing and image processing and VLSI. 